\title{
Dependence of LTX plasma performance on surface conditions as determined by in situ analysis of plasma facing components
}

\author{
M. Lucia ${ }^{\text {a*, R. Kaita }}{ }^{\mathrm{a}}$, R. Majeski ${ }^{\mathrm{a}}$, F. Bedoya ${ }^{\mathrm{b}}$, J.P. Allain ${ }^{\mathrm{b}}$, T. Abrams ${ }^{\mathrm{a}}$, R.E. Bell ${ }^{\mathrm{a}}$, D.P. \\ Boyle $^{a}$, M.A. Jaworski ${ }^{a}$ J.C. Schmitt ${ }^{\mathrm{a}}$ \\ ${ }^{a}$ Princeton Plasma Physics Laboratory (PPPL), Princeton, NJ 08543, USA \\ ${ }^{b}$ University of Illinois at Urbana-Champaign (UIUC), Urbana, IL 61801, USA
}

\begin{abstract}
The Materials Analysis and Particle Probe (MAPP) diagnostic has been implemented on the Lithium Tokamak Experiment (LTX) at PPPL, providing the first in situ x-ray photoelectron spectroscopy (XPS) surface characterization of tokamak plasma facing components (PFCs). MAPP samples were exposed to argon glow discharge conditioning (GDC), lithium evaporations, and hydrogen tokamak discharges inside LTX. Samples were analyzed with XPS, and alterations to surface conditions were correlated against observed LTX plasma performance changes. Argon GDC caused the accumulation of nm-scale metal oxide layers on the PFC surface, which appeared to bury surface carbon and oxygen contamination and thus improve plasma performance. Lithium evaporation led to the rapid formation of a lithium oxide $\left(\mathrm{Li}_{2} \mathrm{O}\right)$ surface; plasma performance was strongly improved for sufficiently thick evaporative coatings. Results indicate that a $5 \mathrm{~h}$ argon GDC or a $50 \mathrm{~nm}$ evaporative lithium coating will both significantly improve LTX plasma performance.
\end{abstract}

PACS: 52.55.Hc, 52.70.-m, 79.60.-i

PSI-21 keywords: XPS, Glow discharge conditioning, Lithium coating, LTX

*Corresponding author address: PPPL, P.O. Box 451, Princeton, NJ 08543-0451, USA

*Corresponding authore-mail: mlucia@pppl.gov

Presenting Author: Matthew Lucia

Presenting author e-mail: mlucia@pppl.gov 


\section{Introduction}

Understanding the interplay between surface conditions and plasma performance is a key aspect in the continuing advancement of magnetic confinement fusion research. Indeed, various metrics of tokamak plasma performance are affected by such plasma facing component (PFC) surface conditions as elemental composition, chemical state, and physical morphology [1]. Plasma performance in modern fusion devices is typically well-analyzed with a variety of plasma diagnostic systems, but surface condition analysis is often limited to a qualitative narrative of wall-conditioning techniques—baking, glow discharge conditioning (GDC), boronization, etc. In this light, surface analysis techniques can provide a quantitative and analytic foundation for describing surface condition.

Past experiments have attempted either to reconstruct the surface history of an ex situ campaign-integrated PFC sample following air exposure [2] or else to reproduce tokamak conditions in offline experiments [3]. This work details the first in situ x-ray photoelectron spectroscopy (XPS) surface analysis of a tokamak PFC. This analysis has been accomplished via the Materials Analysis and Particle Probe (MAPP) diagnostic [4][5], a collaborative effort between PPPL and UIUC. MAPP is designed for sample surface analysis on timescales as brief as one plasma discharge.

For this study, MAPP's XPS analysis capability was used to study samples exposed to argon GDC, lithium evaporations, and hydrogen plasma discharges in the Lithium Tokamak Experiment (LTX) at PPPL. Experiments sought to correlate the nanoscale changes in PFC surface composition observed with MAPP XPS to the macroscopic changes in LTX plasma performance observed with LTX's suite of more traditional plasma diagnostics. Such a correlation would ideally inform the optimization of LTX wall conditioning techniques going forward. 


\section{Materials and methods}

LTX is a spherical torus magnetic confinement device $\left(R_{0}=40 \mathrm{~cm}, a=26 \mathrm{~cm}\right)$ [6][Fig. 1(a)]. Plasma discharges are limited by four conducting toroidal quadrants, creating a shell conformal to the plasma. The shell quadrants (hereafter, "shells") are composed of 0.16 $\mathrm{cm}$ 304L stainless explosively bonded to $0.95 \mathrm{~cm}$ OFHC copper, providing a combination of good lithium compatibility and high thermal conductivity. Up to $\mu \mathrm{m}$-scale lithium coatings can be applied evaporatively to the shells, providing a lithium PFC over $75 \%$ of the plasma's edge. Two toroidal breaks, two poloidal breaks, and eight mid-shell penetrations allow for diagnostic access. The shells are independently heatable to $325{ }^{\circ} \mathrm{C}$, allowing for operation with a liquid lithium PFC. LTX base pressure is typically $5 \times 10^{-8}$ Torr, mostly $\mathrm{H}_{2}$ and $\mathrm{N}_{2}$, but it transiently exceeds $10^{-4}$ Torr due to hydrogen fueling with each plasma discharge. Lithium evaporation is accomplished by a two-step process. Liquid lithium is first dropped onto a pre-heated $\left(300{ }^{\circ} \mathrm{C}\right)$ lower shell via a hole in the bottom of a resistively heated crucible, forming a macroscopic pool of lithium on the shell bottom. An electron beam is then used to heat the pool above $450^{\circ} \mathrm{C}$.

The MAPP head carries four small $(d=0.94 \mathrm{~cm})$ interchangeable sample disks, and it is mounted on a bellows drive so that it can be inserted into the LTX plasma chamber [Fig. 1(c)] and then retracted into a private analysis chamber [Fig. 1(b)], which can be sealed and

pumped independently. MAPP base pressure is typically $5 \times 10^{-8}$ Torr, mostly $\mathrm{H}_{2} \mathrm{O}$ and $\mathrm{CO}_{2}$, rising as high as $1 \times 10^{-7}$ Torr when biasing the $\mathrm{x}$-ray source filament. This present work used MAPP's XPS capability to measure elemental composition and chemical state information of the sample surfaces. For XPS, MAPP houses a PSP Vacuum TX400 x-ray source and a Comstock AC-901 electrostatic energy analyzer. Samples were grounded to LTX machine ground. Energy calibration was performed using single-point reference either to $\mathrm{Au}-4 \mathrm{f}$ from a gold sample or to $\mathrm{C}-1 \mathrm{~s}$ or F-1s from some adventitious surface component, as available. 
Atomic fractions for elemental composition were calculated as a ratio of peak areas above a Shirley background, accounting for empirical elemental sensitivity factors [7].

\section{Argon glow discharge cleaning}

\subsection{Experimental}

Gold and 316 stainless MAPP samples were exposed to two argon glows inside LTX. Glows were struck with an electrode inserted through the outboard toroidal break and biased 600 VAC against grounded shells. Neutral argon pressure was 3 mTorr, and the glow appeared visibly uniform throughout the volume between shells. For both exposures, the MAPP head was located flush with the shells through the outboard toroidal break [Fig. 1(c)].

The first glow (hereafter, "pre-lithium glow") occurred prior to any lithium evaporation after machine venting and cleaning. The glow lasted $13 \mathrm{~h}$ spaced over several days, but MAPP was only exposed for a continuous $1.5 \mathrm{~h}$, with XPS scans completed immediately before and after exposure. LTX plasma discharges were conducted before and after the glow to gauge its effect on plasma performance.

The second glow (hereafter, "post-lithium glow") occurred following three months of occasional lithium evaporation on three of the shells, altogether totaling $\sim 1 \mathrm{~g}$. Glow duration was $5.2 \mathrm{~h}$, and in this case MAPP was exposed for the entire time. XPS scans were completed immediately before and after the glow exposure, and intermediate survey scans were taken $0.3 \mathrm{~h}$ and $1.5 \mathrm{~h}$ into the glow.

\subsection{Results}

Following the pre-lithium glow, both the gold and 316 stainless samples acquired a 12 \pm 2 at. $\% \mathrm{Fe}$ and $6 \pm 1$ at. $\% \mathrm{Cr}$ signature. These elements were not previously present on the 316 stainless sample surface due to a sufficiently-thick adventitious carbon layer. At the same 
time, the Au-4f signal disappeared from the gold sample. Both surfaces gained a significant amount of oxygen, and the Fe-2p peak structure of each suggested both reduced and oxidized iron, respectively at $707 \mathrm{eV}$ and $710 \mathrm{eV}$.

Compared to pre-glow discharges, post-glow discharges lasted more than twice as long and had almost triple the plasma current [Fig. 2]. Spectroscopic C II and O II filterscopes both recorded less total emission from plasma discharges after the glow, but no direct comparison of impurity concentration is possible because temperature data was not available for these discharges.

Following the post-lithium glow, all MAPP samples had acquired a coating of lithium and oxygen thick enough that no other elements were visible. The intermediate survey scans reveal that the coating built up progressively during the entire $5.2 \mathrm{~h}$ period. A second peak appears in both the $\mathrm{C}-1 \mathrm{~s}$ and $\mathrm{O}-1 \mathrm{~s}$ regions, attributable to $\mathrm{Li}-\mathrm{C}-\mathrm{O}$ and $\mathrm{Li}-\mathrm{O}$ bonding, while the $\mathrm{C}-\mathrm{C}$ bonding peak in the $\mathrm{C}-1 \mathrm{~s}$ region almost completely disappears [Fig. 3].

\subsection{Discussion}

Glow discharge cleaning has a long history as a wall-conditioning method in magnetic confinement fusion devices, and the plasma performance improvement following the prelithium glow demonstrates its effectiveness in LTX. H.F. Dylla reports that an argon glow ion fluence of $1 \times 10^{23} \mathrm{~m}^{-2}$ completely removed surface carbon contamination from stainless [8]. In this work, the MAPP sample surface carbon was halved after the $1.5 \mathrm{~h}$ pre-lithium glow, and it was almost completely absent after the $5.2 \mathrm{~h}$ post-lithium glow. Exact LTX glow parameters are unknown, but assuming a typical glow discharge with $T_{e}=5 \mathrm{eV}$ and $n_{i}=1 \times$ $10^{16} \mathrm{~m}^{-3}$, argon ion fluence would be $4 \times 10^{23} \mathrm{~m}^{-2}$ for the pre-lithium glow (only $5 \times 10^{22} \mathrm{~m}^{-2}$ for MAPP exposure portion) and $2 \times 10^{23} \mathrm{~m}^{-2}$ for the post-lithium glow. These values are in good agreement with Dylla. 
However, physical sputtering of carbon as the sole wall conditioning mechanism is not consistent with the observed accumulation of iron, chromium, and oxygen on both gold and 316 stainless MAPP samples. In addition, W. Eckstein calculates a threshold energy of $70 \mathrm{eV}$ for argon on carbon, but only $20 \mathrm{eV}$ for argon on iron and chromium [9]; the $70 \mathrm{eV}$ ion energy is not possible in a glow discharge, but the $20 \mathrm{eV}$ ion energy should be available from an argon glow's $5 T_{e}$ sheath potential. Thus, the argon should only sputter the metal components of the LTX shell, although trace hydrogen and oxygen impurities might be available to chemically sputter the carbon. The resulting wall conditioning mechanism would be the re-deposition of a thin $\mathrm{Fe}_{2} \mathrm{O}_{3} / \mathrm{Cr}_{2} \mathrm{O}_{3}$ layer everywhere on the surface of the LTX shells; remaining carbon impurities would thus be sequestered, and oxygen impurities would be bound in the metal oxide. This would account for the better plasma performance because fewer impurities would enter the plasma, as reflected in the reduced plasma C II and O II filterscope signals.

In fact, the stoichiometry of the MAPP results is consistent with a thin $\mathrm{Fe}_{2} \mathrm{O}_{3}$ and $\mathrm{Cr}_{2} \mathrm{O}_{3}$ layer accounting for $40 \%$ of the XPS signal distributed uniformly over the now-buried original carbon-dominated surface, which still accounts for the remaining $60 \%$ of the XPS signal. Given the first-order attenuation law for photoelectron signal [10], the thickness $d$ of such an ideal uniform layer can be calculated:

$$
\begin{aligned}
& I(z)=I_{0} \exp \left[\frac{-z}{\lambda_{X} \cos \theta}\right] \\
& d=\lambda_{m} \cos \theta \log \left[1+\left(\frac{\lambda_{c}}{\lambda_{m}}\right)\left(\frac{S_{m}}{S_{c}}\right)\right]
\end{aligned}
$$

where $S$ is the signal from the carbon/metal oxide, $\lambda$ is the photoelectron inelastic mean free path of the carbon/metal oxide, and $\theta$ is the angle of the energy analyzer from the surface normal. For the relevant parameters here, $d=0.3 \mathrm{~nm} \approx 1 \mathrm{ML}$. With the assumption that glow-induced sputtering and re-deposition occurs uniformly over LTX, a sputter yield can be 
estimated as the ratio of $\mathrm{Fe} / \mathrm{Cr}$ atoms on the MAPP samples to total Ar fluence: $Y_{A r} \rightarrow F e, C r=1$ $\times 10^{-4}$, which is at least consistent with sputter yields just above the threshold energy.

A similar calculation is possible for the post-lithium glow, where MAPP results suggest that lithium is sputtered from the LTX shells and re-deposited around the vessel. With the different parameters, $d=2.0 \mathrm{~nm} \approx 7 \mathrm{ML}$ and $Y_{A r_{\rightarrow} L i}=4 \times 10^{-4}$. Future work should diagnose plasma discharges immediately before and after a post-lithium glow in order to determine the effect of these lithium re-deposition levels on LTX plasma performance.

\section{Lithium evaporation and passivation}

\subsection{Experimental}

LTX shell (copper-backed 304L stainless) and TZM molybdenum alloy MAPP samples were exposed to three electron beam evaporations from the lower shell lithium pool. In each case, the MAPP head was located flush with the shells through the outboard toroidal break, so the evaporation from the pool was line-of-sight to the samples. XPS scans were completed immediately before and after each evaporation. Estimates of lithium evaporation rate were possible via measurements from a back-shell thermocouple directly beneath the lithium pool. Given the lithium vapor pressure curve $P_{L i}\left(T_{L i}\right)$, total evaporated mass was given by Langmuir evaporation:

$$
\Gamma_{v a p}\left(T_{L i}\right)=\frac{P_{L i}\left(T_{L i}\right)}{\sqrt{2 \pi m_{L i} T_{L i}}}
$$

yielding $3 \pm 1 \mathrm{mg}, 100 \pm 40 \mathrm{mg}$, and $80 \pm 30 \mathrm{mg}$ for the three evaporations. Based on the geometry of lithium pool and MAPP head, these masses were converted to MAPP sample coating thicknesses: $1.6 \pm 0.4 \mathrm{~nm}, 60 \pm 20 \mathrm{~nm}$, and $50 \pm 20 \mathrm{~nm}$, respectively.

The first evaporation (hereafter, "2-nm evaporation") was a 6 min beam run at 0.80 $\mathrm{kW}$ beam power on a $260{ }^{\circ} \mathrm{C}$ pre-heated lower shell. No argon glows, lithium evaporations, or plasma discharges had occurred in LTX for $46 \mathrm{~d}$ prior to this evaporation. Afterward, LTX 
was operated for 10 plasma discharges to determine the evaporation's effect on plasma performance.

The second evaporation (hereafter, "60-nm evaporation") was a 5 min beam run at $1.30 \mathrm{~kW}$ power followed (after a 20 min duty cycle break) by a 6 min beam run at $1.45 \mathrm{~kW}$ power, both on a $310{ }^{\circ} \mathrm{C}$ pre-heated lower shell. Following this evaporation, LTX was operated for 13 plasma discharges.

The third evaporation (hereafter, "50-nm evaporation") was a 6 min beam run at 1.45 $\mathrm{kW}$ beam power on a $320{ }^{\circ} \mathrm{C}$ pre-heated lower shell. This evaporation marked the beginning of a 3 d passivation experiment. LTX was operated for 2 plasma discharges (to estimate reproducibility) at each of $2 \mathrm{~h}, 20 \mathrm{~h}, 41 \mathrm{~h}$, and $70 \mathrm{~h}$ after the evaporation. XPS scans were performed immediately before and after each pair of plasma discharges to determine if the plasma was also altering the surface.

\subsection{Results}

Following the 2-nm evaporation, a lithium signature appeared on both LTX shell and TZM MAPP samples; however, it was not strong enough to completely attenuate the signals from the substrate metals. The carbon/oxygen ratio dropped significantly after the evaporation, and the lithium/oxygen ratio settled around two. Post-evaporation plasma performance was comparable to that with bare stainless shells: Discharges lasted 9-10 ms, with plasma current peaking at 8-10 kA and average density peaking at $1.7-2.1 \times 10^{18} \mathrm{~m}^{-3}$. Triple Langmuir probes had been installed on the centerstack and lower shell, and these measured edge electron temperatures of $15-20 \mathrm{eV}$ and 6-8 eV, respectively.

The 60-nm and 50-nm evaporations coated the LTX shell sample with enough lithium that the substrate metal signals were completely attenuated. The carbon signature was also greatly suppressed, particularly in the 50-nm evaporation, while the lithium/oxygen ratio 
settled around two within a few hours of evaporation and remained unchanged during the three-day passivation test. Plasma performance improved strongly following the $60-\mathrm{nm}$ evaporation [Fig. 4], with $38-40 \mathrm{~ms}$ discharges that peaked at 52-54kA plasma current and 2.3-2.7 $\times 10^{18} \mathrm{~m}^{-3}$ average density. The centerstack and lower shell triple probes measured a slightly hotter edge plasma, at $20-25 \mathrm{eV}$ and $10-15 \mathrm{eV}$, respectively. In comparison with historical filterscope data from passivated lithium walls, the post-evaporation C II and O II emission was reduced by an order of magnitude, but the Li I and Li II emission was increased by an order of magnitude.

Plasma performance was not strongly changed in the $70 \mathrm{~h}$ period following the $50-\mathrm{nm}$ evaporation [Fig. 4]. Discharge plasma current reached the same maximum, but decayed slightly more quickly, resulting in a 3-4ms shorter discharge. Average plasma density ramped up more quickly, but it never reached a higher maximum due to the shorter length of discharges.

\subsection{Discussion}

The 60-nm and 50-nm evaporations saturate the XPS probe depth, but MAPP results from the 2-nm evaporation can be used to benchmark against the validity of the thermocouple evaporation model. In this evaporation, the average substrate metal (Fe, $\mathrm{Cr}, \mathrm{Mo}$ ) was attenuated $89 \pm 1 \%$. A slight reworking of (2) allows calculation of the required lithium layer for the observed attenuation: $d=2.3 \pm 0.1 \mathrm{~nm}$. Given neglected potential errors in the geometrical conversion factor and in the assumption of a uniform coating, the agreement with the model is quite good.

Despite a wide variation in lithium coating thickness among the three evaporations, the surface lithium-oxygen ratio always tended to about two, suggesting the formation of $\mathrm{Li}_{2} \mathrm{O}$. While $\mathrm{LiOH}$ is favored in bulk reaction between lithium and water, various studies 
have reported a preference for $\mathrm{Li}_{2} \mathrm{O}$ in high vacuum [3][11]. J.R. Hoenigman and R.G. Keil have observed $\mathrm{Li}_{2} \mathrm{O}$ forming exclusively up to $10^{2} \mathrm{~L} \mathrm{H}_{2} \mathrm{O}$ exposure and preferentially up to $10^{3} \mathrm{~L} \mathrm{H}_{2} \mathrm{O}$ exposure [12]; for the current cold-shell $P_{\mathrm{H}^{2} \mathrm{O}}=2 \times 10^{-9}$ Torr in LTX, these doses respectively correspond to $14 \mathrm{~h}$ and to $6 \mathrm{~d}$. Interestingly, this latter time period coincides somewhat with the observed rate of post-lithium decay for LTX plasma performance. This suggests that $\mathrm{Li}_{2} \mathrm{O}$ is still capable of pumping hydrogen ions, while $\mathrm{LiOH}$ is not. A similar but longer passivation experiment should be performed, lasting at least two weeks.

\section{Conclusions}

Surface analysis results from argon GDC imply that a $2 \mathrm{~nm}$ surface layer of metal (Fe, $\mathrm{Cr}$, or $\mathrm{Li}$ ) oxide effectively sequesters surface carbon impurity. For plasma performance considerations, this indicates optimum argon glow duration of $10 \mathrm{~h}$ on bare stainless shells and $5 \mathrm{~h}$ on lithium-coated shells. Plasma performance demonstrates substantial improvement with $50 \mathrm{~nm}$ evaporative lithium coatings, but not with $2 \mathrm{~nm}$ coatings. Accordingly, lithium evaporation should be performed with the lower shell pre-heated to near maximum temperature and with the electron beam run at near maximum power. The discrepancy here between an effective $2 \mathrm{~nm}$ glow-induced shell coating and an ineffective $2 \mathrm{~nm}$ evaporationinduced shell coating requires further study. Uniformity of lithium coating coverage may be the key difference; the argon GDC appeared to re-deposit the lithium very uniformly, while the beam evaporation coats only two shell quadrants.

\section{Acknowledgements}

This work is supported by U.S. DOE contracts DE-AC02-09CH11466, DE-AC5207NA27344, and DE-SC0010717. This material is based upon work supported by the National Science Foundation Graduate Research Fellowship under Grant No. DGE-0646086. 


\section{References}

[1] H.W. Kugel et al., Phys. Plasmas 15, 056118 (2008)

[2] C.N. Taylor et al., J. Appl. Phys. 109, 053306 (2011)

[3] R. Sullenberger, MS thesis, Princeton University, 2012.

[4] C.N. Taylor et al., Rev. Sci. Instrum. 83, $10 \mathrm{D} 703$ (2012)

[5] M. Lucia et al., Rev. Sci. Instrum. 85, 11D835 (2014)

[6] R. Majeski et al., Phys. Plasmas 20, 056103 (2013)

[7] D. Briggs and M.P. Seah, eds. Practical Surface Analysis, Vol. 1. John Wiley \& Sons, 2e.: New York, 1992.

[8] H.F. Dylla, J. Vac. Sci. 6 (3), 1276-1287 (1988)

[9] R. Behrisch and w. Eckstein, eds. Sputtering by Particle Bombardment. Springer:

Berlin, 2007.

[10] L.A. Giannuzzi and F.A. Stevie. Introduction to focused ion beams instrumentation, theory, techniques, and practice. Springer, 1e.: New York, 2005.

[11] H. Sugai et al., J. Nucl. Mater. 220-222 (1995) 254-258

[12] J.R. Hoenigman and R.G. Keil, App. Surf. Sci. 18 (1984) 207-222

\section{Figure captions}

Fig. 1 - (a) A cutaway projection of LTX; (b) MAPP head in analysis position; (c) MAPP head in exposure position.

Fig. 2 - Comparison of LTX plasma performance immediately before (red) and immediately after (blue) the pre-lithium argon GDC. 
Fig. 3 - Changes observed in C-1s and O-1s peaks of 316 stainless MAPP sample, with traces from immediately before (red), 2 hours after (blue), 11 hours after (black), and 8 days after (magenta) the post-lithium argon GDC.

Fig. 4 - Comparison of LTX plasma performance immediately before (red), immediately after (blue), and 3 days after (black) the 50-nm lithium evaporation.

\section{Figures}

Figure 1

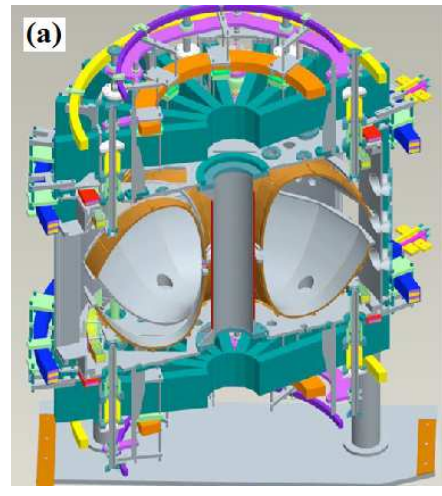

(b)
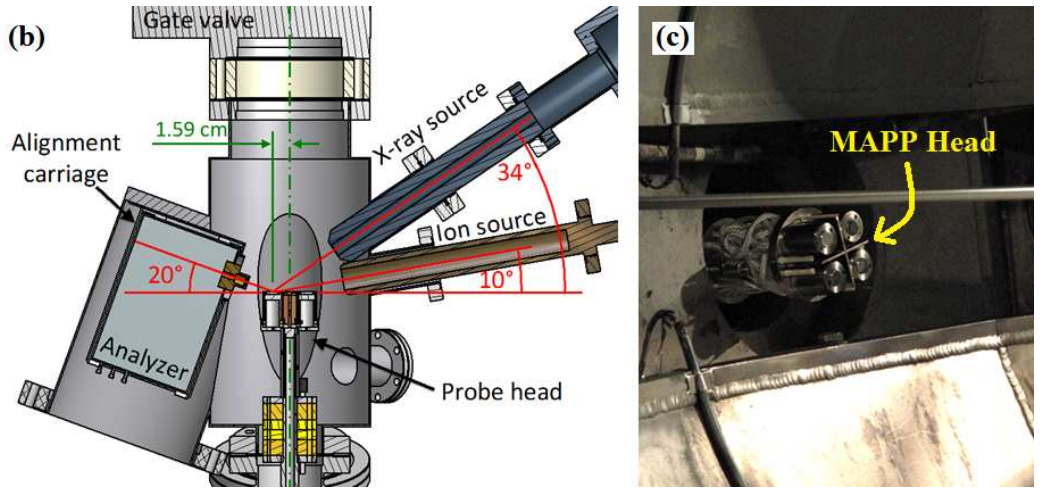
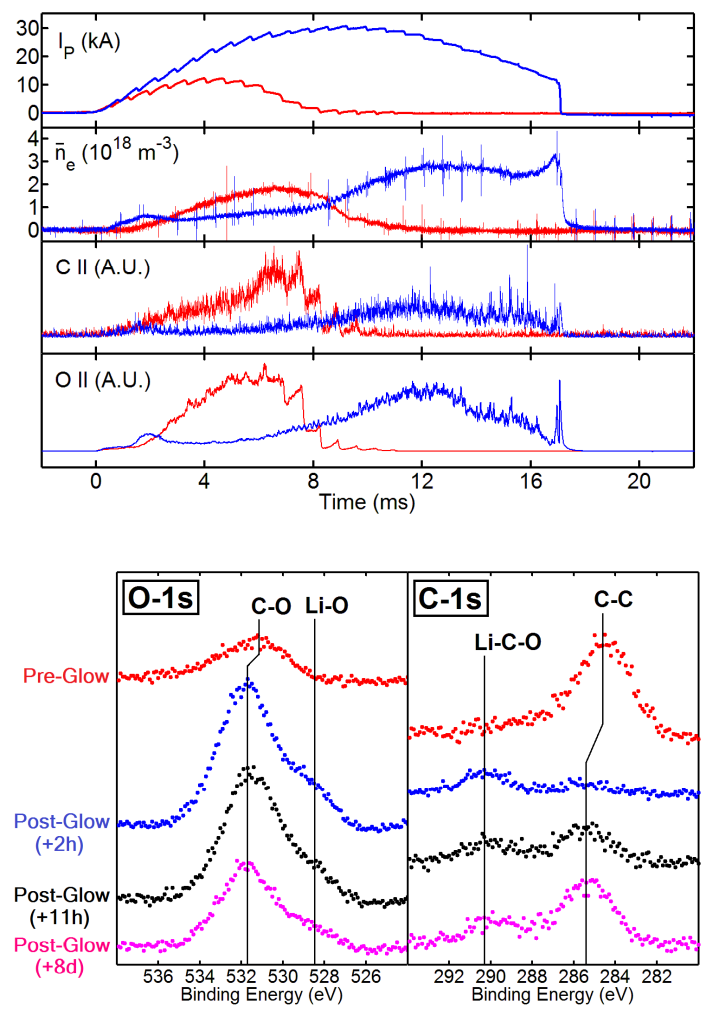

Figure 2

Figure 3 


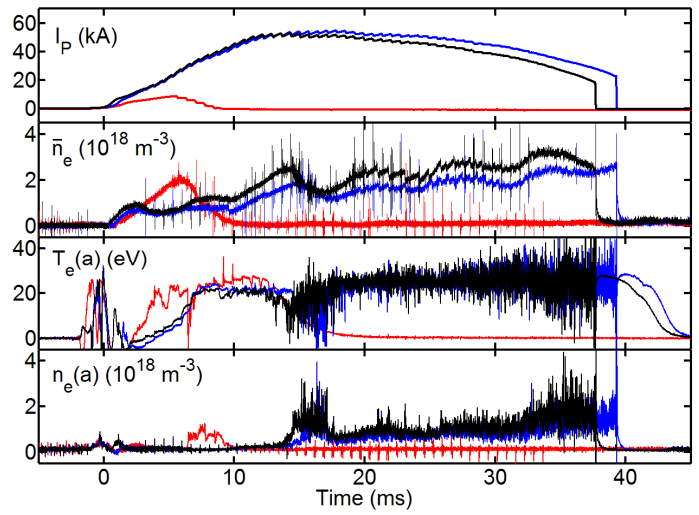

Figure 4 